\title{
Phenotypical screening on metastatic PRCC-TFE3 fusion translocation renal cell carcinoma organoids reveals potential therapeutic agents
}

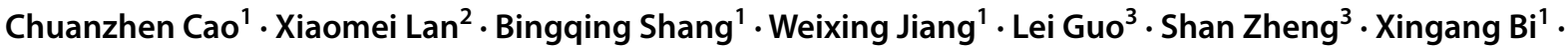 \\ Aiping Zhou ${ }^{4} \cdot$ Zhijian Sun $^{2} \cdot$ Jianzhong Shou ${ }^{1}$ (])
}

Received: 30 September 2021 / Accepted: 31 December 2021 / Published online: 3 February 2022

(c) The Author(s) 2022

\begin{abstract}
Purpose Translocation renal cell carcinoma (tRCC) is a subtype that occurs predominantly in children and young individuals. Metastatic tRCC occurring in young patients is more aggressive than that occurring in older patients, and there are still no effective therapies. Organoids can mimic original tissues and be assessed by high-throughput screening (HTS). We aimed to utilize patient-derived organoids and HTS to screen drugs that can be repurposed for metastatic tRCC with PRCC-TFE3 fusion.

Methods Tumor tissues were obtained from treatment-naïve metastatic tRCC patients who underwent surgery. Histopathology and fluorescence in situ hybridization (FISH) confirmed the tRCC. Organoids derived from the dissected tissues were cultured and verified by FISH and RNA-seq. HTS was performed to seek promising drugs, and potential mechanisms were explored by RNA-seq and cell-based studies.

Results We successfully established a metastatic tRCC organoid with PRCC-TFE3 fusion, a common fusion subtype, and its characteristics were verified by histopathology, FISH, and RNA-seq. An HTS assay was developed, and the robustness was confirmed. A compound library of 1816 drugs was screened. Eventually, axitinib, crizotinib, and JQ-1 were selected for further validation and were found to induce cell cycle arrest and apoptosis. RNA-seq analyses of posttreatment organoids indicated that crizotinib induced significant changes in autophagy-related genes, consistent with the potential pathogenesis of tRCC.

Conclusions We established and validated organoids derived from tissues dissected from a patient with metastatic tRCC with PRCC-TFE3 fusion and achieved the HTS process for the first time. Crizotinib might be a targeted therapy worthy of exploration in the clinic for metastatic tRCC with PRCC-TFE3 fusion. Such organoid and HTS assays may represent a promising model system in translational research assisting in the development of clinical strategies.
\end{abstract}

Keywords Translocation renal cell carcinoma $\cdot$ Metastatic $\cdot$ Organoid $\cdot$ High-throughput screening

Aiping Zhou

zhouaiping_cicams@yeah.net

$\triangle$ Zhijian Sun

zjsun@k2oncology.com

$\triangle$ Jianzhong Shou

shoujzh2021@163.com

1 Department of Urology, National Cancer Center/National Clinical Research Center for Cancer/Cancer Hospital, Chinese Academy of Medical Sciences and Peking Union Medical College, Panjiayuan Nanli 17\#, Chaoyang District, Beijing 100021, People's Republic of China
2 K2 Oncology Co. Ltd., Beijing 100176, People's Republic of China

3 Department of Pathology, National Cancer Center/National Clinical Research Center for Cancer/Cancer Hospital, Chinese Academy of Medical Sciences and Peking Union Medical College, Beijing 100021, People's Republic of China

4 Department of Medical Oncology, National Cancer Center/National Clinical Research Center for Cancer/Cancer Hospital, Chinese Academy of Medical Sciences and Peking Union Medical College, Panjiayuan Nanli 17\#, Beijing 100021, People's Republic of China 


\section{Introduction}

Microphthalmia transcription factor (MiT) family translocation renal cell carcinoma (tRCC) is a subtype of kidney cancer that occurs predominantly in children and adolescents but only accounts for $1.3 \sim 1.5 \%$ of adult RCCs [ 1 , 2]. It has been recently defined as a distinct subset of RCC classified by characteristic morphology and clinical presentation. The unique characteristic of tRCC is the fusion of various partner genes with transcription factor E3 (TFE3), which is located on chromosome Xp11.2 [3]. The common fusion types in tRCC include PRCC-TFE3, ASPL-TFE3, and PFS-TFE3 [4]. The mechanisms underlying the oncogenic roles of TFE3 fusions in tRCC have not yet been elucidated. Previous studies indicated that TFE3 fusions could regulate autophagy and lysosomal biogenesis, which were involved in oncogenic signaling [5]. As the activity of the promoters of fusion-affected genes increases, the overexpression of fusion proteins potentiates the intrinsic oncogenic features of MiT-TFE transcription factors [3].

Pediatric tRCC is mostly indolent, while tRCC diagnosed in young adults is more aggressive with unfavorable outcomes [6,7]. Adult patients with metastatic tRCC also present a poorer prognosis than those with non-tRCC disease [8]. However, there is no accepted standard therapy for metastatic tRCC. Therefore, understanding the functions of TFE3 fusion as an intrinsic pro-oncogenic driver might reveal potential drug targets.

Organoids are in vitro culture models of human tissues; they have a high success rate in terms of model generation and can be established quickly and retain the original tissue structure and biological information. Organoids hold great promise in the exploration and screening of potential treatment options. Over the past years, patient-derived organoids (PDOs) have been established and are becoming crucial models in basic research and drug discovery. For kidney cancer, however, only a few studies using patientderived materials such as primary cell lines or tumor cellderived organoids have been published about drug screening, and these studies did not involve tRCC $[9,10]$.

High-throughput screening (HTS) is an automated process that allows for the rapid testing of large chemical, genetic, or biological libraries. It utilizes microplates, liquid handling devices, sensitive detectors, and data-processing software to identify a small number of effectors of a particular biological mechanism from libraries.

Here, we describe the establishment of the first organoid for metastatic tRCC with a common fusion (PRCC-TFE3) and describe the HTS process with a goal of discovering effective drugs in vitro that could be further evaluated in the clinic.

\section{Materials and methods}

\section{Human tumor tissues}

All experiments with human tissue were approved by the Ethics Committee of the National Cancer Center/Cancer Hospital, Chinese Academy of Medical Sciences (NCC/ CHCAMS) (ID: 20/246-2442), and written informed consent was obtained. Renal tumor tissue was obtained from treatment-naïve patients who underwent nephrectomy at the Department of Urology, NCC/CHCAMS. The sample was processed within $24 \mathrm{~h}$ after surgery. Moreover, pathological evaluation and TFE3 break-apart fluorescence in situ hybridization (FISH) detection confirmed the malignancy of the samples and the TFE3 fusion status.

\section{PDO culture}

On arrival, tumor tissue was extensively washed with cold phosphate buffered saline (PBS) and then minced into cubes smaller than $1 \mathrm{~mm}^{3}$ on ice. Subsequently, the minced tissue pieces were digested with collagenase $(2 \mathrm{mg} / \mathrm{ml}$, Cat. No. C9407, Sigma-Aldrich, St Louis, MO, USA) for $1 \mathrm{~h}$ at $37^{\circ} \mathrm{C}$ and filtered through sieves with $100-\mu \mathrm{m}$ pores. After the samples were washed twice with fresh medium ( $2 \%$ fetal calf serum, FCS; advanced DMEM/F 12, 2 mM HEPES, $1 \times$ GlutaMAX-I, $200 \mathrm{U} / \mathrm{ml}$ penicillin/streptomycin) and centrifugation ( $300 \mathrm{~g}, 5 \mathrm{~min})$, the dissociated cells were seeded into growth factor-reduced Matrigel (Corning Inc., Corning, NY, USA) in the presence of advanced $\mathrm{DMEM} / \mathrm{F} 12$ at $37{ }^{\circ} \mathrm{C}$ for $30 \mathrm{~min}$. Next, the surface of solidified mixture of cell suspension/Matrigel was sealed with complete human organoid medium (HOM, $1 \mathrm{ml}$ ), which was comprised of Advanced DMEM/F12 supplementing with series additives $(1 \times \mathrm{B} 27$ supplement, $10 \mathrm{nM}$ Lue15Gastrin I, $1 \mathrm{mM} \mathrm{N}$-acetylcysteine, $100 \mathrm{ng} / \mathrm{ml}$ recombinant human IGF-1, $50 \mathrm{ng} / \mathrm{ml}$ recombinant FGF-2, 20\% afamin/ Wnt 3 a conditional medium, $1 \mu \mathrm{g} / \mathrm{ml}$ human R-spondin, $100 \mathrm{ng} / \mathrm{ml}$ Noggin, $500 \mathrm{nM}$ A-8301, $200 \mathrm{U} / \mathrm{ml}$ penicillin/streptomycin, and $10 \mu \mathrm{M}$ Y-27632) as described by Lampis et al. [11] and Loredana et al. [12]. The medium was replaced every 3 days when the diameter of organoids ranged up to $200 \sim 300 \mu \mathrm{m}$, and the organoids were dissociated and passaged weekly. Matrigel was broken up by pipetting up and down several times, and organoids were collected in a tube. After centrifugation at $300 \mathrm{~g}$ for 5 min, organoids were dissociated using TrypLE Express (Gibco, Grand Island, NY, USA) for $10 \mathrm{~min}$. Cell clusters were reseeded at a typical split ratio of 1:3. A brightlight microscope (LEICA, Wetzlar, Germany) was used for organoid bright field photographing. Early passage 
organoids were frozen in Recovery Cell Culture Freezing Medium (Gibco) and stored at $-80^{\circ} \mathrm{C}$ before drug screening. The whole process from tissue collection to compound screening is shown in Fig. 1A. A detailed protocol will be provided by the authors upon request.

\section{Histology, immunohistochemistry, and TFE3 break-apart FISH assay}

Tumor tissues and organoids were fixed in 4\% paraformaldehyde followed by dehydration, paraffin embedding, sectioning and standard hematoxylin and eosin (H\&E)
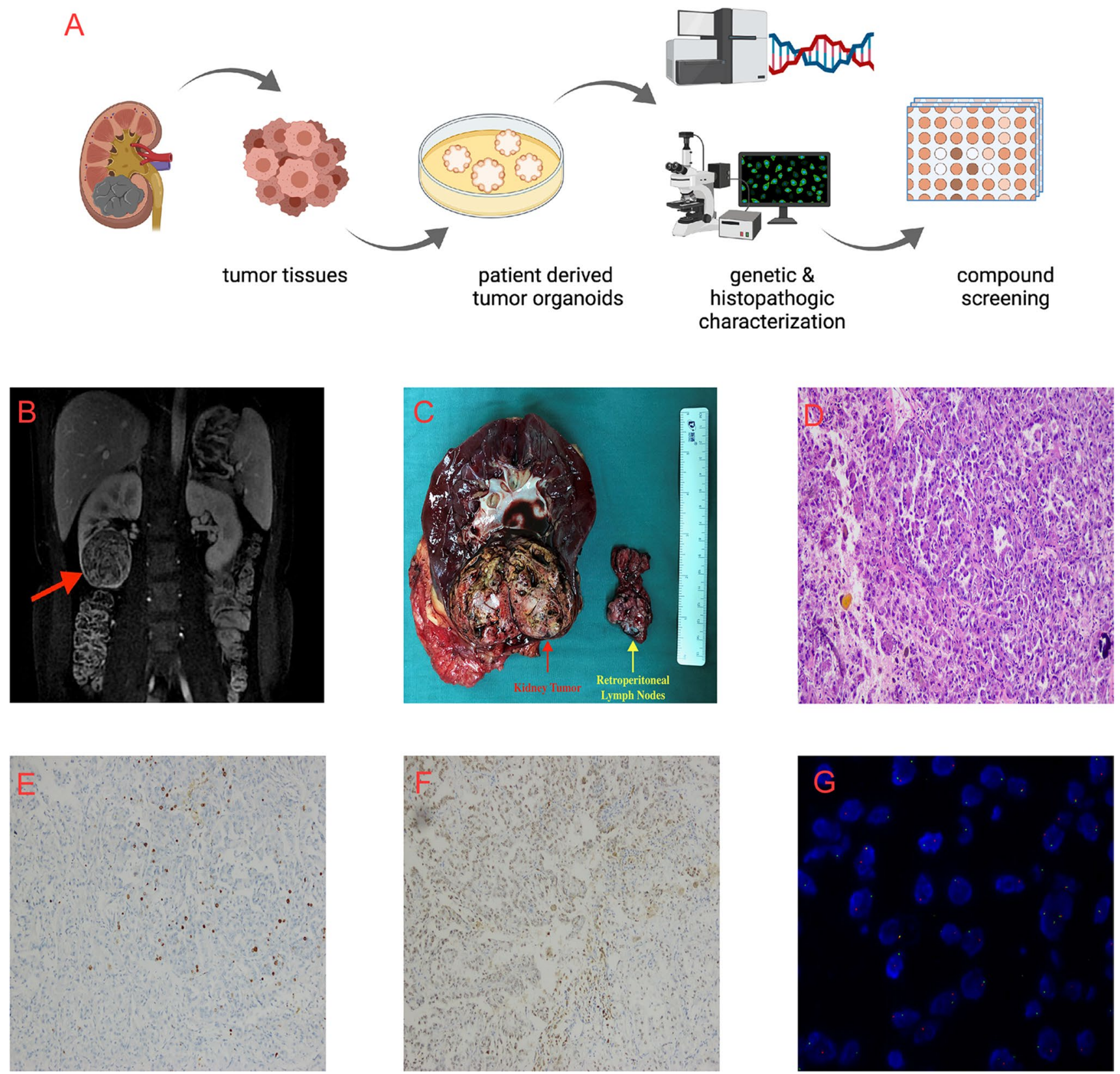

Fig. 1 Schematic illustration and clinical feature of the metastatic PRCC-TFE3 fusion tRCC case. A The whole process from tissue obtain to compound screening (created by BioRender). B MRI indicates a solid tumor of $6.0 \times 5.0 \times 5.2 \mathrm{~cm}$ located at the lower pool of right kidney with arterial phase enhancement. $\mathbf{C}$, The specimen picture of right kidney with a tumor located at the lower pool and the retroperitoneal lymph nodes. D H\&E staining slide at $100 \times$ magnification of renal cell carcinoma showed eosinophilic staining of tumor cytoplasm, papillary structure, foci of necrosis, and gravel-like calcification. E and F Immunohistochemistry of Ki-67 (5\%) and TFE3 protein (positive) at $100 \times$ magnification. G TFE3 break-apart FISH assay indicated the cells with split-signals accounted for $70 \%$. Signals were considered to be split when the green and red signals were separated by a distance $\geq 2$ signal diameters. Normal cells of male showed one signal of yellow while TFE3 fusion tRCC cells showed two split signals of green and red 
staining. Immunohistochemistry (IHC) staining was performed with TFE3 (dilution-free. RMA-0663, Shandong Maixin Biotechnology Co., China), E-cadherin (dilution 1: 200. MAB-0738, Shandong Maixin Biotechnology Co., China), and Ki-67 (dilution 1: 200. GM7240, GeneTech Co., Ltd., Shanghai, China) antibodies on an Autostainer 480S (Fa Medac). Images of stained samples were taken with an Olympus DP73 microscope (Olympus, Japan).

TFE3 protein expression confirmed by IHC is routinely used for preliminary diagnosis, but clinically FISH is the gold standard for diagnosis of TFE3 fusion tRCC [13]. A dual-color, break-apart FISH assay was performed to detect TFE3 using the TFE3 (Xp11.2) break probe set (Guangzhou LBP Medical Technology Co., Ltd., China). FISH signals were assessed under an Olympus BX51TRF microscope (Olympus, Japan). Normal cells without fusion had one (for males) or two (for females) yellow signals. Signals were considered to be split when the distance between red and green signals was $\geq 2$ signal diameters. For each case, a minimum of 100 tumor nuclei were evaluated. Overlapping cells indistinguishable as separate nuclei were not included in the analysis to avoid false positives due to nuclear truncation. A positive result was defined when $\geq 15 \%$ of the tumor nuclei had split signals.

\section{Compound library screening}

HTS was performed automatically using a mechanical liquid handling equipment mechanical arm (NAYO N96-204, NAYO Biotech. Co., Ltd. Shanghai, China). A drug library containing 1816 small molecule compounds was obtained from MedChemExpress (Shanghai, China, catalog number HY-L0022M) with modification (Supplementary Data 1). The library was reformatted into 96-well source plates with a concentration of $1 \mathrm{mM}$ for automated robotic screening. In parallel, the cells were also treated with an equal volume $(0.2 \%)$ of dimethyl sulfoxide (DMSO) as a negative control and 2.5 $\mu \mathrm{M}$ final BEZ-235 (MCE, Shanghai, China) as a positive control. BEZ-235 is an inhibitor of phosphatidylinositol 3-kinases (PI3-K) and is commonly used as a positive control in HTS or drug exploration [14, 15]. Plate-to-plate normalization and assay quality control were performed according to them. A 3D cell viability assay was implemented to determine the number of viable cells according to ATP level using commercially available luminescence detection reagent (CellTiter-Glo \#G9683, Promega, Madison, WI). Briefly, organoids were processed as described previously [16] and plated in a 96-well low binding assay plate at a density of 8,000 cells per well in $50 \mu 110 \%$ growth factor reduced Matrigel. Additional $40 \mu \mathrm{l}$ culture medium without Matrigel was added as described above. Organoids were maintained in medium described earlier [17] and treated 2 days later by adding $10 \mu \mathrm{l}$ culture medium with
$10 \mu \mathrm{M}$ compound to obtain a final concentration of $1 \mu \mathrm{M}$. The assay was terminated at day 5 by adding $50 \mu \mathrm{l}$ CellTiterGlo. Assay quality and robustness were evaluated with the signal window (SW) and Z factor. The SW and the $\mathrm{Z}$ factor have been routinely used in previous HTS studies for validation [18-20]. Triplicate wells treated with BEZ-235 and vehicle solvent (DMSO) were employed as bottom wells and top wells, respectively. When SW was above 6 and the $\mathrm{Z}$ factor values were between 0.44 and 0.78 , the assay was qualified for HTS. The equipment of the $\mathrm{Z}$ factor is listed below ( $\sigma$, standard deviation. $\mu$, average. $p$, positive control. $n$, negative control) [21] as follows:

$\mathrm{Z}=1-\frac{3 \times\left(\sigma_{p}+\sigma_{n}\right)}{\left|\mu_{p}-\mu_{n}\right|}$

\section{Analysis of cell cycle arrest and apoptosis}

Cell cycle and apoptosis were detected as previously described [22]. Organoids were cultured and treated with the indicated drugs at different concentrations for $48 \mathrm{~h}$, followed by single staining with propidium iodide (Beyotime Biotechnology Co., Ltd., China) for cell cycle analyses and double staining with propidium iodide and Annexin V-FITC (Beyotime Biotechnology Co., Ltd., China) for apoptosis analyses.

\section{RNA-seq analysis}

Organoids were incubated with DMSO or the indicated drugs for $120 \mathrm{~h}$. After harvesting, total RNA was extracted using the TraZolTM UP Plus RNA Kit. RNA was sent for sequencing and analysis (BGI Co., Ltd., Shenzhen, China). Briefly, total RNA was fragmented, and mRNA was enriched using oligo (dT) magnetic beads, followed by cDNA synthesis. Double-stranded cDNA was purified and enriched by PCR amplification, after which the library products were sequenced using BGIseq-500. The volcano plot and heatmap of differentially expressed genes ( $\log 2$ of fold change $>1, q<0.05$ ) were generated by BGI using the Dr. TOM approach, a customized data mining system from BGI. Next, 232 autophagy-related genes (ARGs) were obtained from the Human Autophagy database (HADb, http://www. autophagy.lu/index.html) and used for analyses.

\section{Statistical analysis}

Data statistical analysis was performed using Prism 6.4. The half-maximal inhibitory concentration $\left(\mathrm{IC}_{50}\right)$ values were analyzed using nonlinear regression (curve fit). The 95\% confidence interval was calculated. The differences 
in $\mathrm{IC}_{50}$ values between organoids were analyzed using the nonparametric Mann-Whitney U test. Cell cycle and apoptosis data were analyzed using Excel with Student's $t$ test. $q<0.05$ was considered to indicate statistical significance.

\section{Results}

\section{Identification of metastatic tRCC patient}

One 21-year-old male patient was diagnosed with right kidney cancer and retroperitoneal lymph node metastasis. Magnetic resonance imaging (MRI) indicated a solid tumor of $6.0 \times 5.0 \times 5.2 \mathrm{~cm}$ located in the lower pool of the right kidney with arterial phase enhancement (Fig. 1B). Laparoscopic nephrectomy and retroperitoneal lymph node dissection were performed in April 2019. The pathology indicated RCC and lymph node metastasis (T1bN0M1, Stage IV) (Fig. 1C). H\&E staining demonstrated that the tumor featured papillary architecture combined with foci of necrosis and gravel-like calcification (Fig. 1D). IHC showed positivity for Ki-67 and TFE3 protein expression (Fig. 1E and F). tRCC was confirmed by the FISH assay (Fig. 1G).

\section{Characterization of PRCC-TFE3 fusion organoids}

After obtaining freshly resected tumor tissue, we successfully established patient-derived tRCC organoids (Fig. 2A and $\mathrm{B})$. The established tRCC organoids were propagated for three or more passages and cryopreserved. H\&E data showed that both organoids and original tissue displayed a cytoplasm papillary structure harboring multiple lumen epithelium folds and invaginations (Fig. 2C). These data demonstrated the association between the morphological structure of tRCC tumors and their derived organoids. IHC staining of Ki-67 (Fig. 2D) and E-cadherin (Fig. 2E) indicated that organoids retained epithelial origin and proliferation activity.

TFE3 break-apart FISH is currently the gold standard for the identification of TFE3 rearrangements [13]. TFE3 break-apart FISH of the derived organoids indicated that the cells with split signals accounted for $69.5 \%$ of the signals (Fig. 2F). Furthermore, RNA-seq was performed, and a fusion of PRCC (exon 1, end with protein IAAPELHKGD) and TFE3 (exon 6, starting with protein CLCQGICLMC) was detected with a frequency of $15 \%$ (Fig. 2G).

Our results indicated that the tissue architecture, phenotype, cellular composition, and typical PRCC-TFE3 gene fusion of the primary tumors were reliably conserved in PRCC-TFE3 fusion organoid models.

\section{Development of a high-throughput phenotypical assay with PRCC-TFE3 fusion organoids}

After successfully establishing and characterizing PRCCTFE3 fusion tRCC organoids, we focused on developing a three-dimensional (3D) cell-based assay that was suitable for HTS. HTS requires robust assays with high reproducibility of well-to-well and plate-to-plate assays, as well as high signal-to-noise ratios. To determine whether PRCC-TFE3 organoids could be seeded and cultured in 3D, we established a workflow employing an automated liquid-handling system (Fig. 3A).

Then, we focused on the validation of assay robustness and reproducibility by assessing plate uniformity using the parameters of SW and Z factor. Three independent experiments were conducted to evaluate the day-to-day variation. In each experiment, 10 plates were used to measure plateto-plate variation. High control and low control wells were defined as organoids treated with medium containing DMSO and BEZ-235. Each treatment well was analyzed in quadruplicate to determine the well-to-well variation. The $\mathrm{Z}$ factors ranged from 0.44 to 0.78 , indicating the signal-to-noise reproducibility between plates was high enough. $\mathrm{Z}$ factors of three independent experiments were $0.61,0.61$, and 0.66 showing the day-to-day variation was low. The average values of for SW and the $\mathrm{Z}$ factor were 6.88 and 0.63 , respectively (Fig. 3B). These results indicated that the assay was qualified for HTS with high reproducibility. Representative images of organoids at different time points are shown in Fig. 3C-J.

\section{High-throughput compound library screening}

To facilitate the screening of thousands of compounds across hundreds of cell lines, we used a small-molecule library containing 1,816 compounds. The target average final concentration of the compound library for screening in cell-based assays was $2 \mu \mathrm{M}$. Most compounds $(1271,70 \%)$ were nononcology-related, and the remaining compounds were either chemotherapeutics or targeted oncology agents.

We designed a 2-stage screening strategy whereby the 1816 drugs were primarily screened in a single well at a single dose $(2 \mu \mathrm{M})$. A total of 101 drugs showed more than $50 \%$ inhibition (Fig. 4A). On the other hand, when analyzing the distribution of activity with the compound category, most compounds were related to epigenetics, tyrosine kinases, the cell cycle and autophagy (Fig. 4B). Interestingly, among the 101 active drugs, most active compounds (61 out of 101 , $60.4 \%$ ) were originally developed for non-oncology clinical indications (Supplementary Fig s1).

As we aimed to use phenotypical drug screening to identify medicines for repurposing and assess potential mechanisms of action for TFE 3 fusion tRCC, 45 chemotherapy 

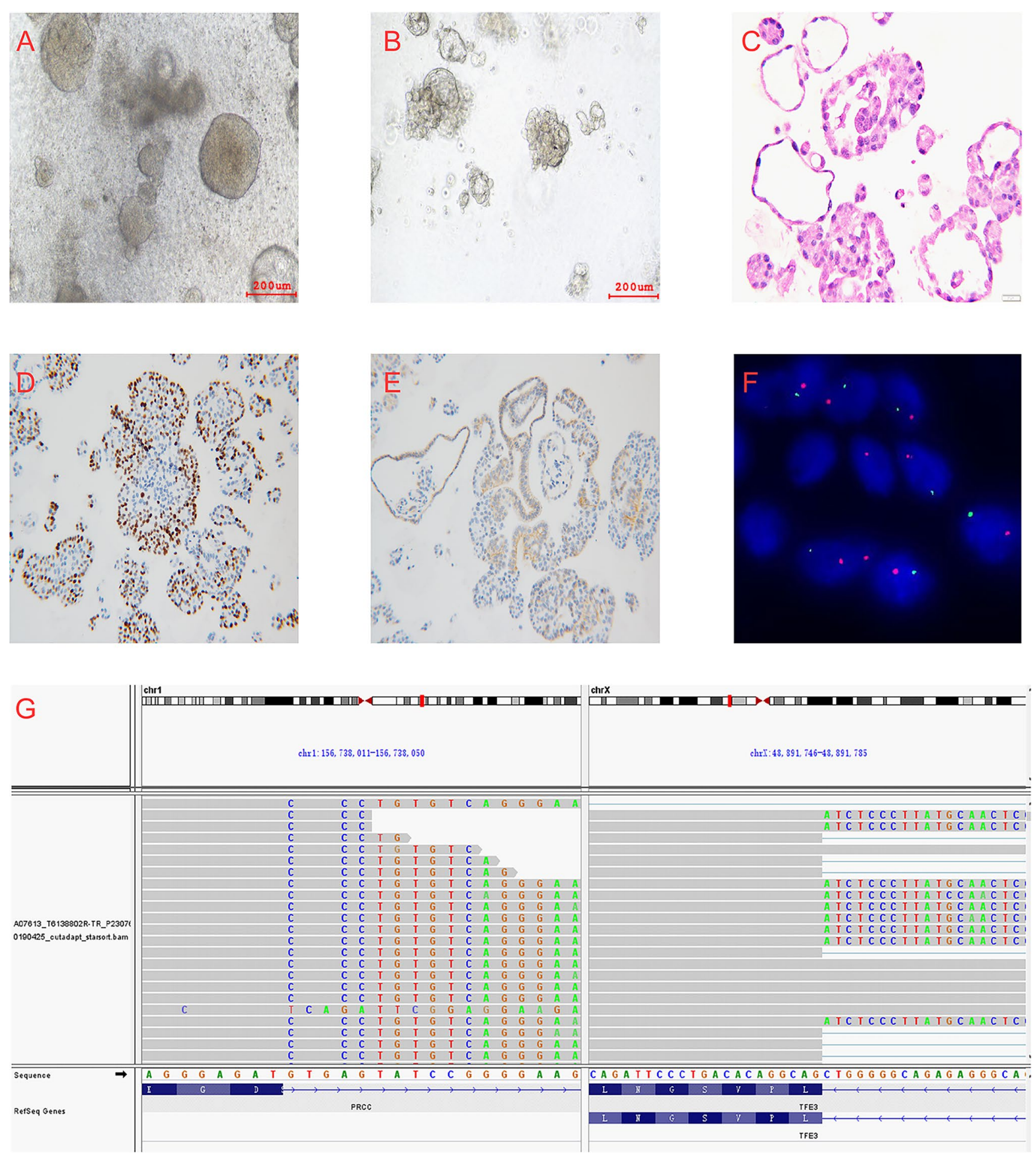

Fig. 2 Characterization of PRCC-TFE3 fusion organoids. A and $\mathbf{B}$ Bright-field images of patient-derived PRCC-TFE3 fusion tRCC organoids in the early stage (passage 0 ) and the later stage (passage 5). C The H\&E staining of the PRCC-TFE3 fusion tRCC organoid showed cystic phenotype with papillary architecture which has similarity with original tumor tissue at $100 \times$ magnification. D Immunohistochemistry of Ki-67 (60\%) at $100 \times$ magnification of the organoid. E Immunohistochemistry of E-cadherin $(>50 \%)$ at $100 \times$ magnifica- tion of the organoid. This demonstrated a clear epithelial morphology of organoids. F, TFE3 break-apart FISH assay indicated the cells with split-signals accounted for $69.5 \%$ in derived organoids, which was highly conserved with original tissue. G, RNA-seq indicated there was $t(X ; 1)(p 11.2 ; q 21)$ resulting in PRCC-TFE3 gene fusion in the organoid by using RNA-seq based on the gene fusion panel (GenomiCare Biotechnology, Shanghai Inc., China) 
A

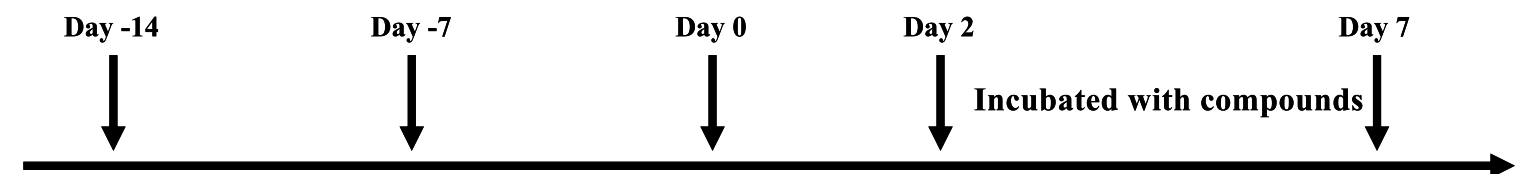

Recovery Medium fed-batch Plating Adding compounds

Cell viability test

B

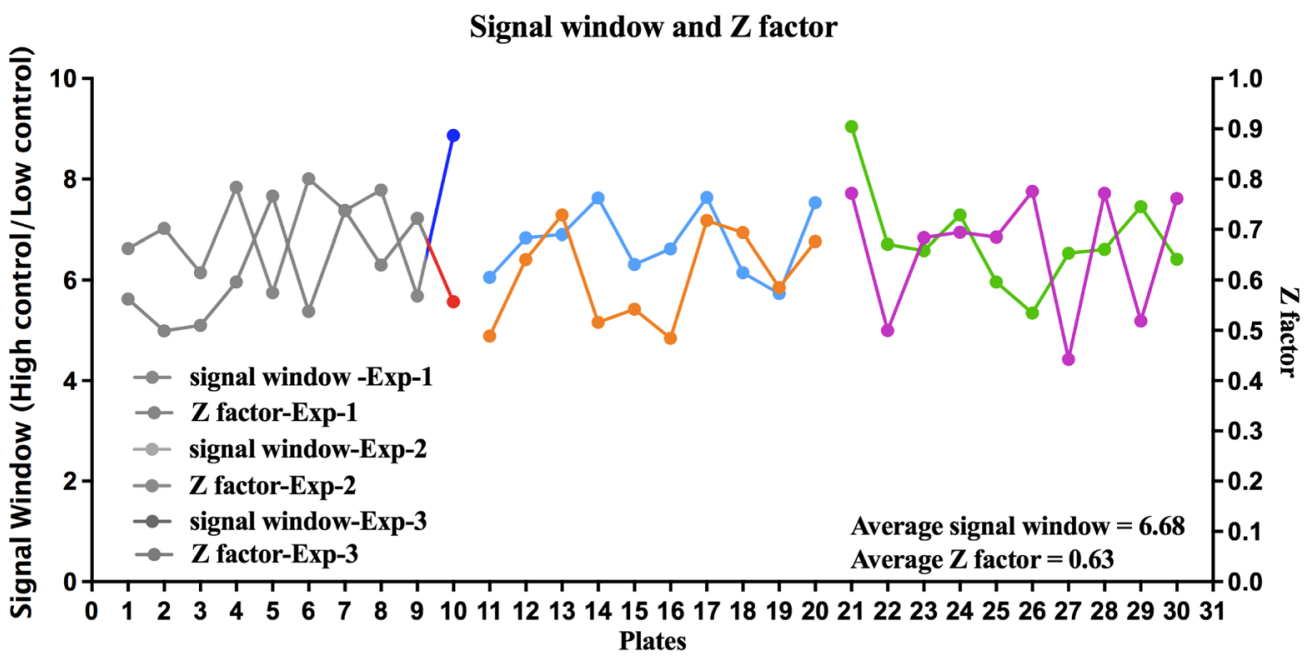

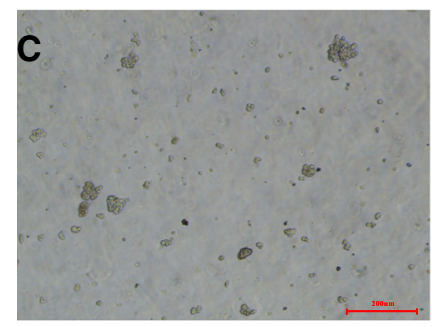
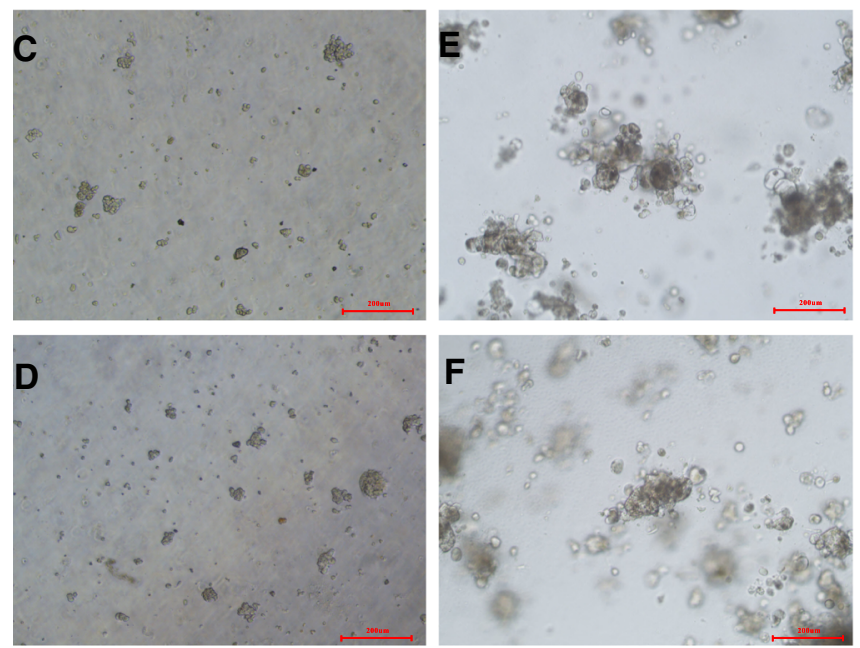

Fig. 3 PRCC-TFE3 fusion tRCC organoids allow high throughput assay in vitro. A Time protocol of the preparation of PDOs and highthroughput drug screening. B Assay quality and robustness were evaluated with signal window (SW) and $\mathrm{Z}$ factor in three independent experiments of ten plates each. BEZ-235 and DMSO were employed as bottom wells and top wells, respectively. The assay showed the

agents with nonspecific cytotoxicity were excluded from the 101 compounds. After that, 56 compounds were retained, and axitinib was manually added to the panel. Eventually, a drug panel containing 57 compounds was designed (Supplementary Data 2) and subjected to testing via an eightpoint dose-response model (doses ranging from $60 \mu \mathrm{M}$ to
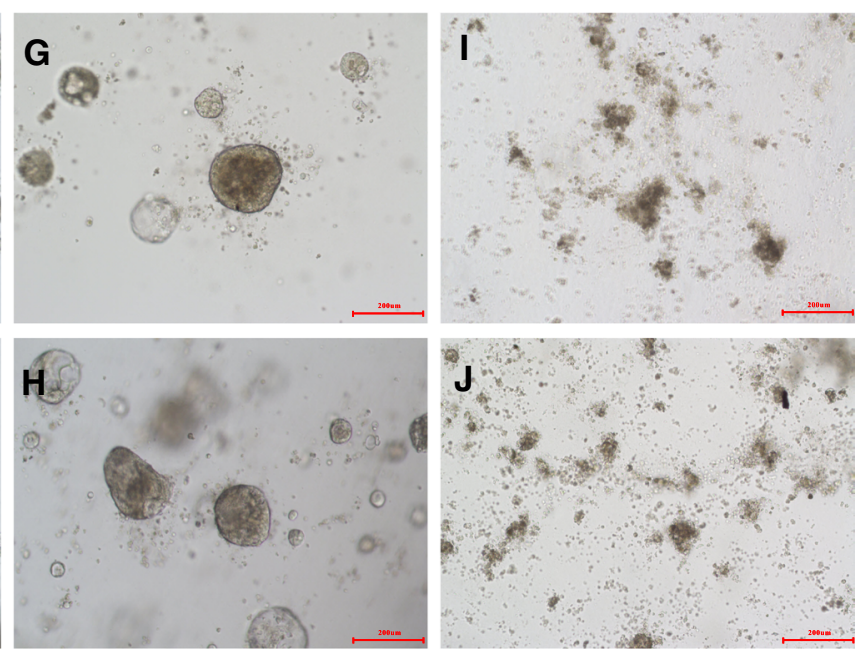

SW was higher than 6 and the $\mathrm{Z}$ factor values were between 0.44 and 0.78 , which indicated the assay was qualified for high-throughput screening. C-J, Representative imaging of organoids at different time points, day $0(\mathbf{C}$ and $\mathbf{D})$, day $2(\mathbf{E}$ and $\mathbf{F})$, day $7(\mathbf{G}$ and $\mathbf{H})$ of DMSO, and day 7 (I and $\mathbf{J}$ ) of BEZ-235

$27 \mathrm{nM}$ ). Finally, three representative drugs, axitinib, crizotinib, and JQ-1, were selected, and the dose-response results indicated that all of them significantly inhibited cell viability (Fig. 4C).

To be noticed, axitinib is a small molecule tyrosine kinase inhibitor that inhibits multiple targets, including vascular 
A
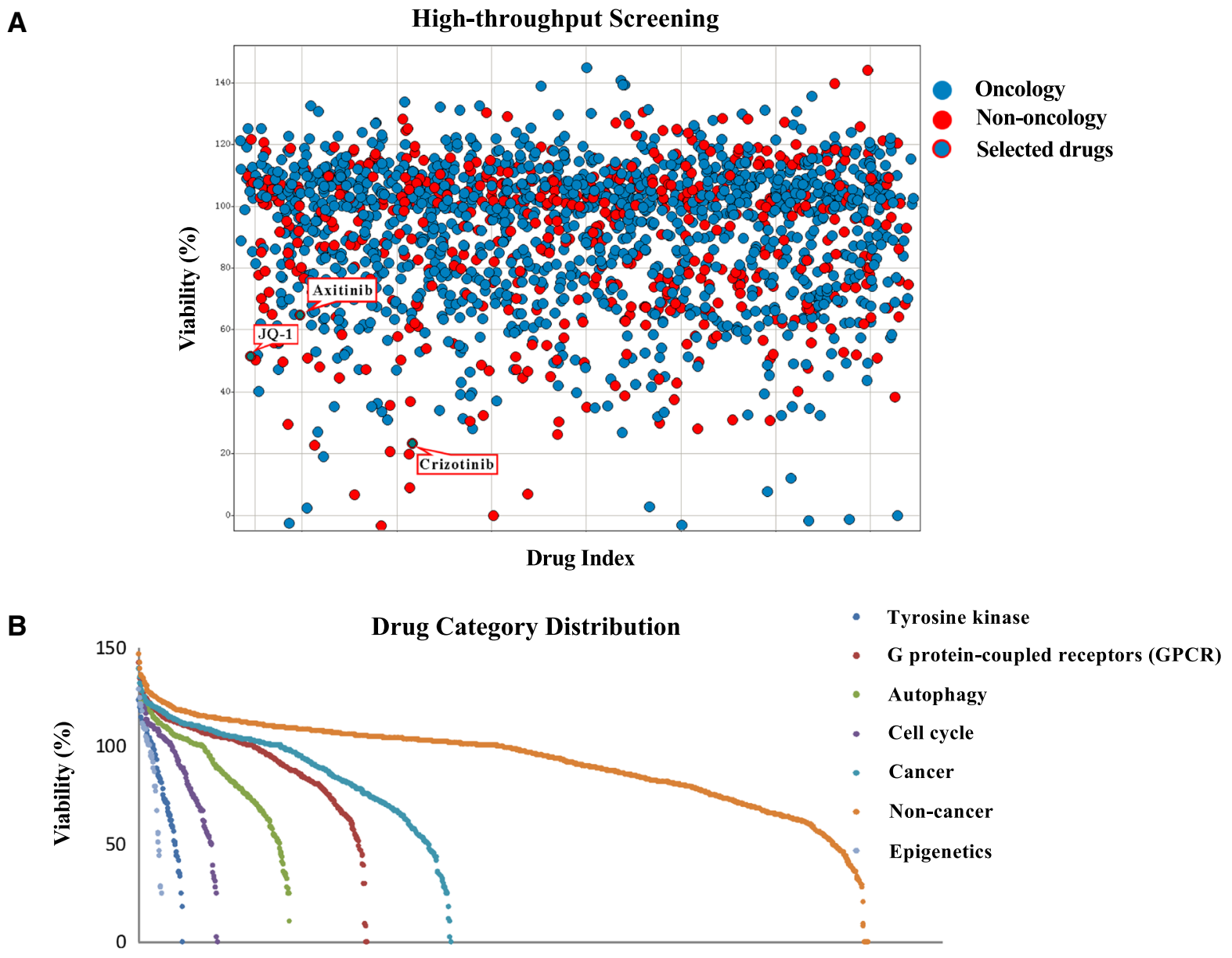

C

Proportion of drugs
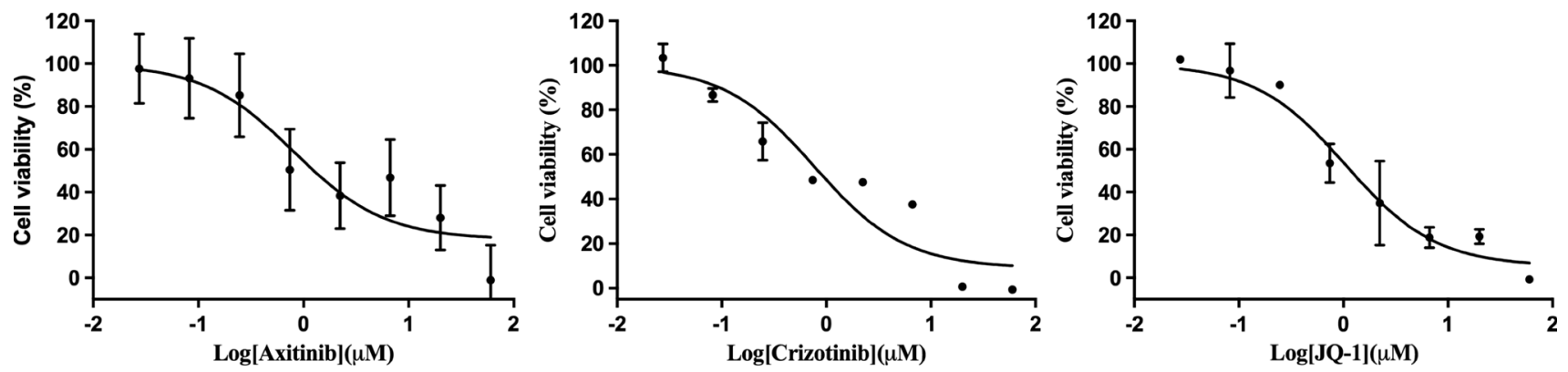

Fig. 4 High throughput screening (HTS) with the compound library. A Primary screening of 1816 compounds in HTS assay. Blue spots represented the original disease was oncology, and red spots represented the original disease was non-oncology. Axitinib, crizotinib, and JQ-1 were identified. B Distribution of activity with compound category including tyrosine kinase, $G$ protein-coupled receptors (GPCR), autophagy-related compounds, cell cycle-related com-

endothelial growth factor receptor. As the PDOs did not contain vascular endothelial cells, axitinib only killed $35.4 \%$ of cell in PRCC-TFE3 fusion tRCC organoids. Axitinib activity in RCC patients has been studied in various settings, particularly as a second-line targeted treatment. Although pounds, cancer, non-cancer, and epigenetics compounds. The epigenetic, tyrosine kinase, cell cycle, and autophagy-related compounds were most frequently effective in viability assay. C The doseresponse curves of axitinib, crizotinib, and JQ-1 in PRCC-TFE3 fusion tRCC organoids. Three selected representative leads were tested in an eight-point dose-response ranging from $60 \mu \mathrm{M}$ to $27 \mathrm{nM}$

axitinib did not reach 50\% inhibition, we also included it for further investigation.

As TFE3 factors in tRCC can regulate autophagy, we then focused on autophagy-related drugs. Autophagy is required for crizotinib-induced apoptosis in MET-amplified gastric 
cancer cells [23]. JQ-1 is a BRD4 inhibitor, and BRD4 is a transcriptional repressor of autophagy and lysosomal function [24]. Therefore, axitinib, crizotinib, and JQ-1 were selected for further investigation.

\section{Cellular mechanism of inhibition of the representative drugs}

To further explore the cellular mechanism of axitinib, crizotinib, and JQ-1 in PDOs, we investigated the cell cycle and apoptosis effects. Proliferation assays demonstrated that all three drugs could inhibit cell proliferation in dose-dependent and time-dependent manners (Fig. 5A-C). The bright-field images $(200 \mu \mathrm{m})$ of tRCC PDOs after incubation of $120 \mathrm{~h}$ with DMSO, axitinib, crizotinib, and JQ-1 showed that compared with the vehicle control (DMSO), three drugs of low-dose or high-dose induced the cell apoptosis significantly (Fig. 5D-F). Fluorescence-activated cell sorting (FACS) showed that axitinib, crizotinib, and JQ-1 induced changes in cell counts during the cell cycle (Fig. 5G). After quantitative analyses, the percentage of cells during the cell cycle showed that JQ-1 induced G0/G1 phase arrest, while axitinib and crizotinib induced G2/M phase arrest (Fig. 5H). All three drugs promoted cell apoptosis, especially early apoptosis, in a dosedependent manner (Fig. 5I), and the cell apoptosis rates with these three drugs were shown in Fig. 5J.

\section{Expression profiling after drug treatment}

To explore the underlying molecular mechanism of the antitumor effect of axitinib, crizotinib, and JQ-1, we performed whole-genome mRNA expression profiling. Total RNA isolated from PDOs treated with axitinib, crizotinib, and JQ-1 was analyzed by 3' mRNA-Seq and compared to that from PDOs treated with DMSO. Using a $\log 2$ of fold change cutoff of 1 and a q-value cutoff of 0.05 , we identified differentially expressed genes. In the axitinib high-dose group, only ten genes were upregulated, and one gene was downregulated. In the JQ-1 high-dose group, eight genes were upregulated, and 28 genes were downregulated. In the crizotinib high-dose group, 364 genes were upregulated, and 671 genes were downregulated $(q<0.05, \log 2$ of fold change $>1)$ (Fig. 6A) (Supplementary Data 3).

We observed that ARGs were modulated by JQ-1 and crizotinib but not by axitinib (Fig. 6B). In particular, crizotinib treatment showed significant effects on ARGs (Fig. 6C) (Supplementary Data 4).

\section{Discussion}

TFE3 fusion tRCC is an aggressive variant when present in young adults. Metastatic tRCC has a poor prognosis, and there are only a few case reports or observational studies on systemic therapy for metastatic tRCC, but the efficacy is neither representative nor ideal [8, 25]. Effective drugs are urgently needed to improve survival. PDOs are promising disease models not only for understanding disease biology but also for exploring drug efficacy in vitro before moving to animal models. The drug screening value of PDOs has been reported in liver cancer, ovarian cancer, and Crohn's disease [26, 27]. In a recent analysis of drug responses in patients, organoid cultures were matched with the patient response up to $90 \%$ [28]. However, there are still no set-up of metastatic tRCC and patient-specific drug screening models. As TFE3 fusion tRCC has unique characteristics of transcription factor translocation, which may become a potential drug target, it is meaningful to explore promising therapeutic drugs for metastatic tRCC by employing PDOs. In our study, we established and characterized the common fusion type of metastatic tRCC PDOs for the first time and successfully performed HTS with 1816 compounds. Crizotinib and JQ-1 had promising curative value in vitro and induced significant gene alterations related to the potential pathogenesis of tRCC. These results reveal novel opportunities for using PDOs for therapeutic drug discovery.

A unique and important feature of PDOs is that they could maintain the mutational landscape of the original tumor tissue even after long-term expansion in culture and upon transplantation into mice for further research. The reproduction of original tumor genetic aberrations in a culture setting makes PDOs a potentially valuable resource in screening drug sensitivity/resistance, identifying novel therapeutics as part of a personalized medicine approach. We successfully established and cultured PDOs of metastatic tRCC with PRCC-TFE3 fusion. Thereafter, we confirmed the close relationship of the PDOs and the tumor tissue of origin by H\&E, IHC, FISH, and RNA-seq assays. The complex structural histology preserved and the presence of TFE3 fusion was validated.

The prognosis of metastatic tRCC in young patients is poor, and currently available targeted therapies do not have great efficacy. In the context of exploring new effective drugs, PDOs are a promising model in the next generation of HTS studies with greater physiological relevance; these studies could involve human species specificity, regenerative applications, and modelling complex phenotypes, but the complexity of organoid cultures poses a significant challenge for miniaturization and automation [29]. Previous organoid culture studies were limited to lineages 

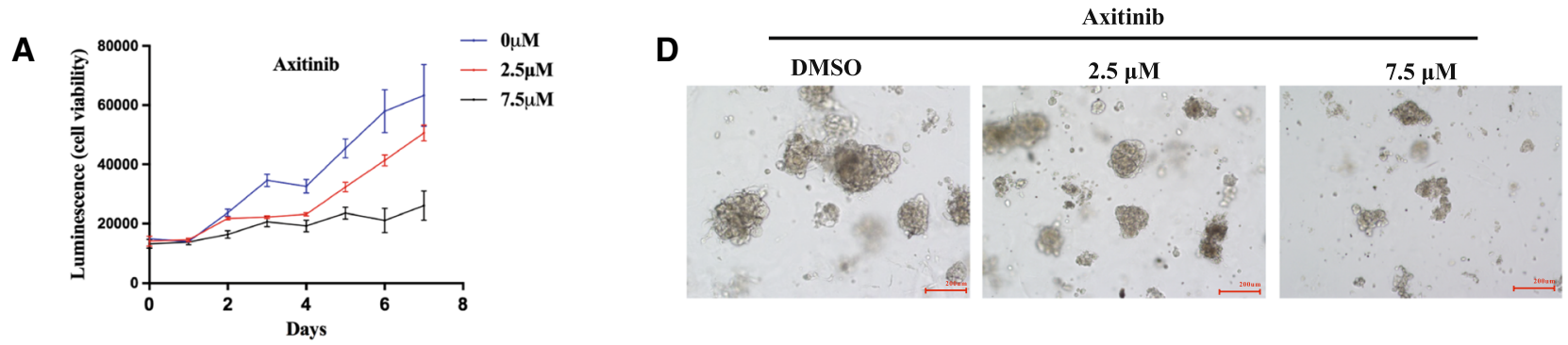

B

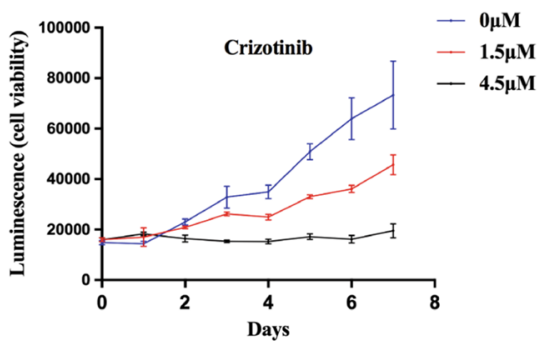

E

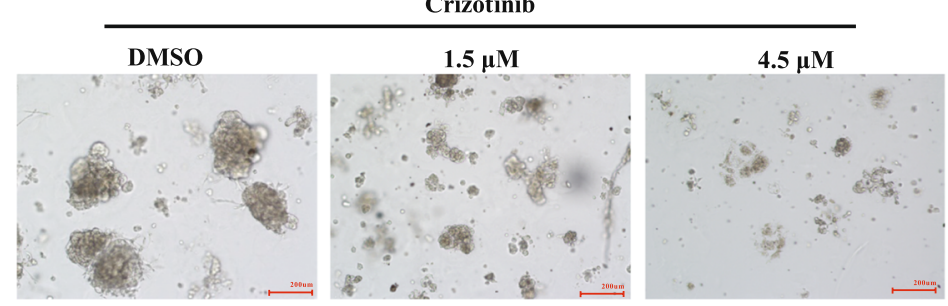

C
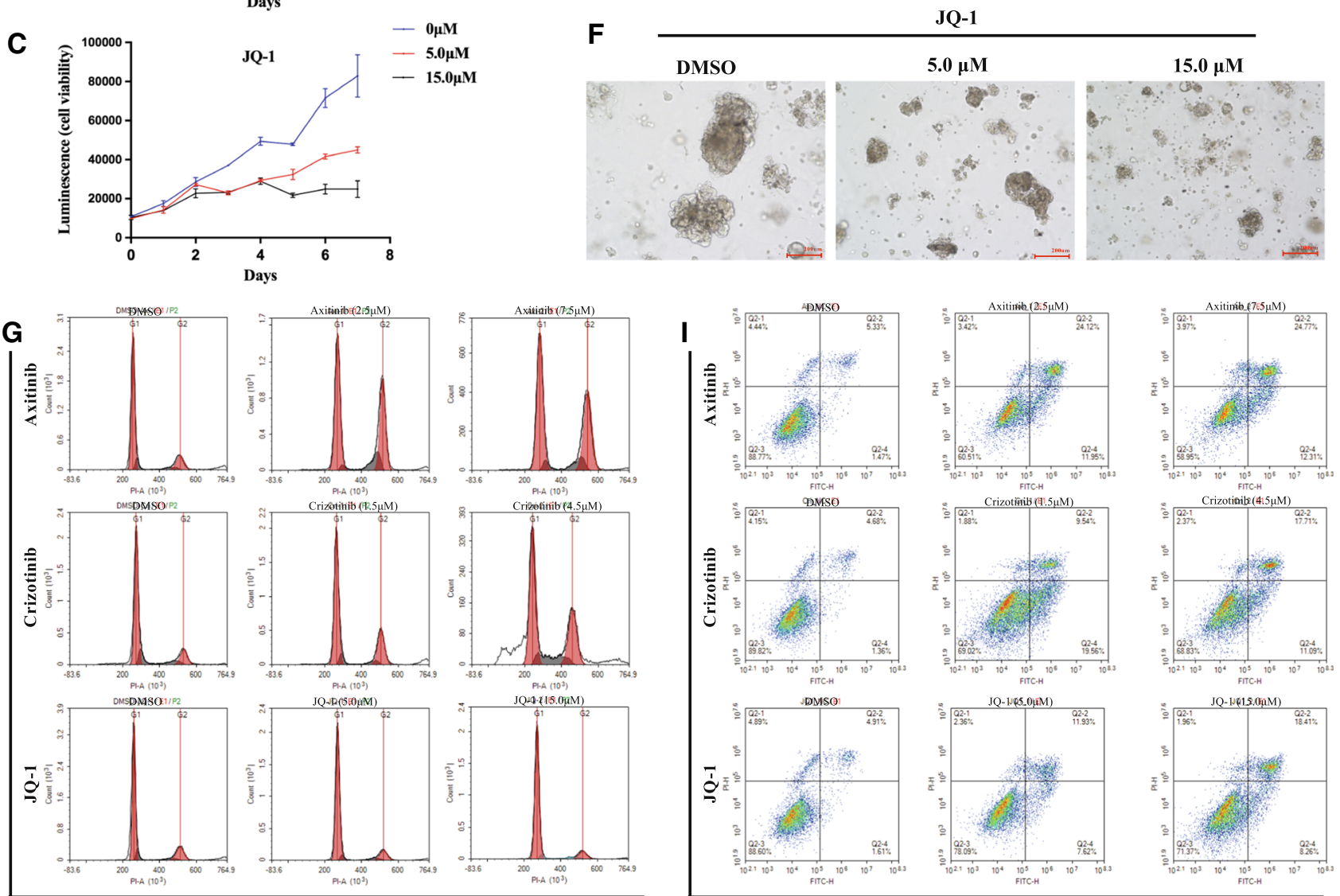

Propidium Iodide
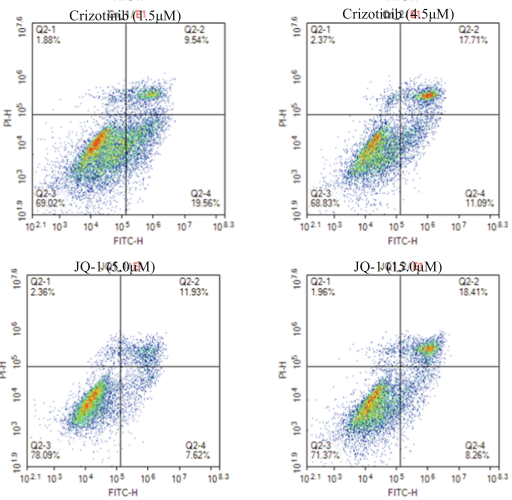

Annexin V-FITC

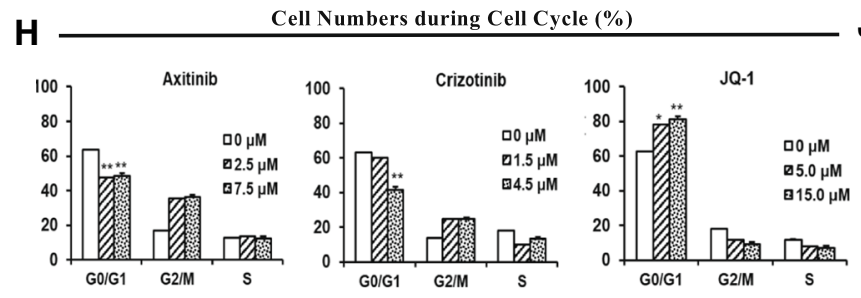

J

Apoptosis (\%)

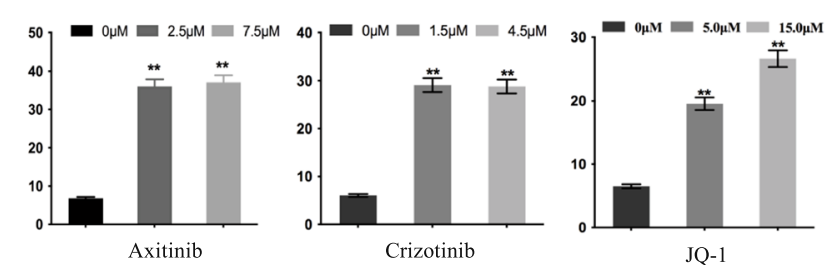


४Fig. 5 Cellular mechanism analyses of axitinib, crizotinib, and JQ-1 in PRCC-TFE3 fusion tRCC PDOs. A-C, axitinib, crizotinib, and JQ-1 dose-dependently suppressed the cell growth in a 7-day proliferation assay. D-F bright-field images $(200 \mu \mathrm{m})$ of tRCC PDOs after incubation of $120 \mathrm{~h}$ with DMSO, axitinib, crizotinib, and JQ-1. Compared with the vehicle control (DMSO), three drugs of low-dose or high-dose induced the cell apoptosis significantly. G fluorescence activating cell sorter (FACS) showed that axitinib, crizotinib, and JQ-1 induced changes in cell counts during the cell cycle. $\mathbf{H}$ after quantitative analyses (One-way Anova) by NovoExpress v1.3.4, the percentage of cell numbers during cell cycles show that JQ-1 induced G0/G1 phase arrest, while axitinib and crizotinib induced G2/M phase arrest. I all three drugs could promote cell apoptosis in dosedependent manner. Cells were treated with DMSO, axitinib $(2.5 \mu \mathrm{M}$, $7.5 \mu \mathrm{M})$, crizotinib $(1.5 \mu \mathrm{M}, 4.5 \mu \mathrm{M})$, and JQ-1 $(5 \mu \mathrm{M}, 15 \mu \mathrm{M})$ for $48 \mathrm{~h}$ and subsequently stained with annexin $\mathrm{v}$ and propidium Iodide for detecting the early stage and late stage of apoptosis. $\mathbf{J}$ the quantitative data of the percentage of apoptosis cells induced by axitinib, crizotinib, and JQ-1

with robust, self-renewing stem cells, such as intestinal crypt cells or mammary tumors. In our study, we established a scalable, HTS-compatible assay for automated generation, maintenance, and optical analysis in standard 96-well plates. In combination with careful evaluation of reproducibility based on well-to-well and plate-to plate assays, these techniques could enable proper interpretation of library-scale drug discovery experiments. To our knowledge, a high-throughput phenotypical assay with tRCC organoids has not previously been generated automatically. Although we focused on PRCC-TFE3 fusionaffected tRCC organoids as a representative lineage, it is likely that the same general techniques could be adapted to produce other fusion types in the future.

In the present study, we found that axitinib, crizotinib, and JQ-1 arrested the cell cycle and induced the apoptosis of PRCC-TFE3 fusion tRCC organoids. Axitinib, a small molecule tyrosine kinase inhibitor, has been adopted as a second-line therapy for clear cell RCC. In addition, a multicenter phase II study with 40 non-clear cell RCCs, including 7 cases of tRCC, indicated that axitinib showed promising efficacy in terms of overall response rate (ORR) and progression-free survival (PFS) as second-line treatment in recurrent or metastatic non-clear cell RCC [30]. Therefore, we also included it for further investigation. Although it did not reach 50\% inhibition during HTS, axitinib indeed induced cell cycle arrest and cell apoptosis, which indicated that it might directly kill tumor cells via other mechanisms. Crizotinib is an ATP-competitive small-molecule inhibitor of the receptor tyrosine kinases C-Met, ALK and ROS1. It inhibited cell growth and induced apoptosis in human gastric carcinoma cell lines in vitro [31]. JQ-1 displays antitumor effects in testicular germ cell tumors, leukemia, and prostate cancer by inhibiting BRD4, and BRD4 is a mediator of transcriptional elongation in the M/G1 cell cycle transition [32].
In our study, we confirmed that axitinib, crizotinib, and JQ-1 indeed promoted cell cycle arrest and apoptosis in a dose-dependent manner.

As the activity of the promoters of TFE3 fusion genes increases, overexpression of fusion proteins potentiates the intrinsic oncogenic features of TFE transcription factors [3]. TFE factors in tRCC can regulate autophagy, lysosomal biogenesis and signaling, which are considerably involved in oncogenic signaling [5]. Furthermore, TFE3 could promote the endolysosomal deprivation reaction by inducing the expression of autolysosomal genes [33, 34]. Recently, Zeng et al. demonstrated that lysosomal and autophagy pathways were significantly upregulated in TFE3-tRCC [35]. Previous studies indicated that elevated doses of crizotinib can induce autophagy in alveolar rhabdomyosarcoma cells [36] and that autophagy is required for crizotinib-induced apoptosis in MET-amplified gastric cancer cells [23]. In fact, the autophagy pathway plays a dual role in tumorigenesis. Recently, Estelle et al. indicated that autophagy has a dual role in cancer development and therapy. It could exert either prosurvival or prodeath functions in anaplastic large cell lymphoma. Differences in autophagy activity lead to differences in treatment efficacy in cancers [37]. In our RNA-seq analysis with ARGs, crizotinib was found to play a role in the antitumor effect in PRCC-TFE3 fusion tRCC organoids. We confirmed the therapeutic value of crizotinib on the PRCC-TFE3 fusion tRCC in vitro. However, the autophagy-related anticancer mechanisms of crizotinib deserve to be explored, and it was promising to further validate crizotinib in animal experiments.

Some limitations warrant discussion. tRCC has several fusion types, and our established PDOs and related drug screening results only represented the PRCC-TFE3 type. However, the PRCC-TFE3 fusion type is one of most common fusion types in tRCC. In addition, the tRCC PDO protocol has been sufficiently developed and can be applied to culture other fusion types in the future. Another limitation is the small number of tRCC PDOs; however, the first PDOs of tRCC might have a certain representative significance.

In conclusion, the organoids derived from metastatic tRCC with PRCC-TFE3 fusion that we presented here fulfilled all the criteria of a reliable in vitro cancer model, recapitulating the dominating features of the most common fusion subtype of tRCC, from histological architecture to genetic traits. The established HTS assay made drug screening amenable. Crizotinib was found to have promising antitumor activity on metastatic tRCC with PRCC-TFE3 fusion in vitro. Such organoids and HTS assays may represent a promising model system for its use both in vitro and in vivo, with a broad range of potential applications in basic and translational research assisting in the development of clinical strategies. 


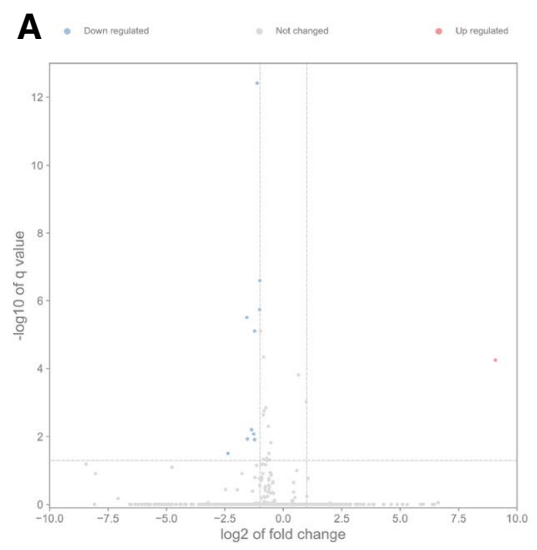

Axitinib (high dose) vs DMSO

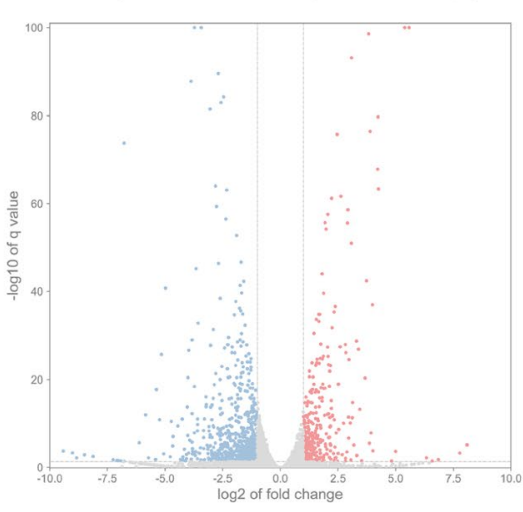

Crizotinib (high dose) vs DMSO

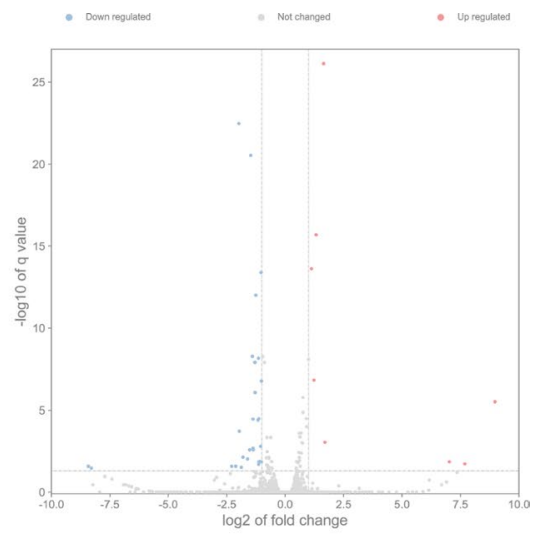

JQ-1 (high dose) vs DMSO

\section{B}
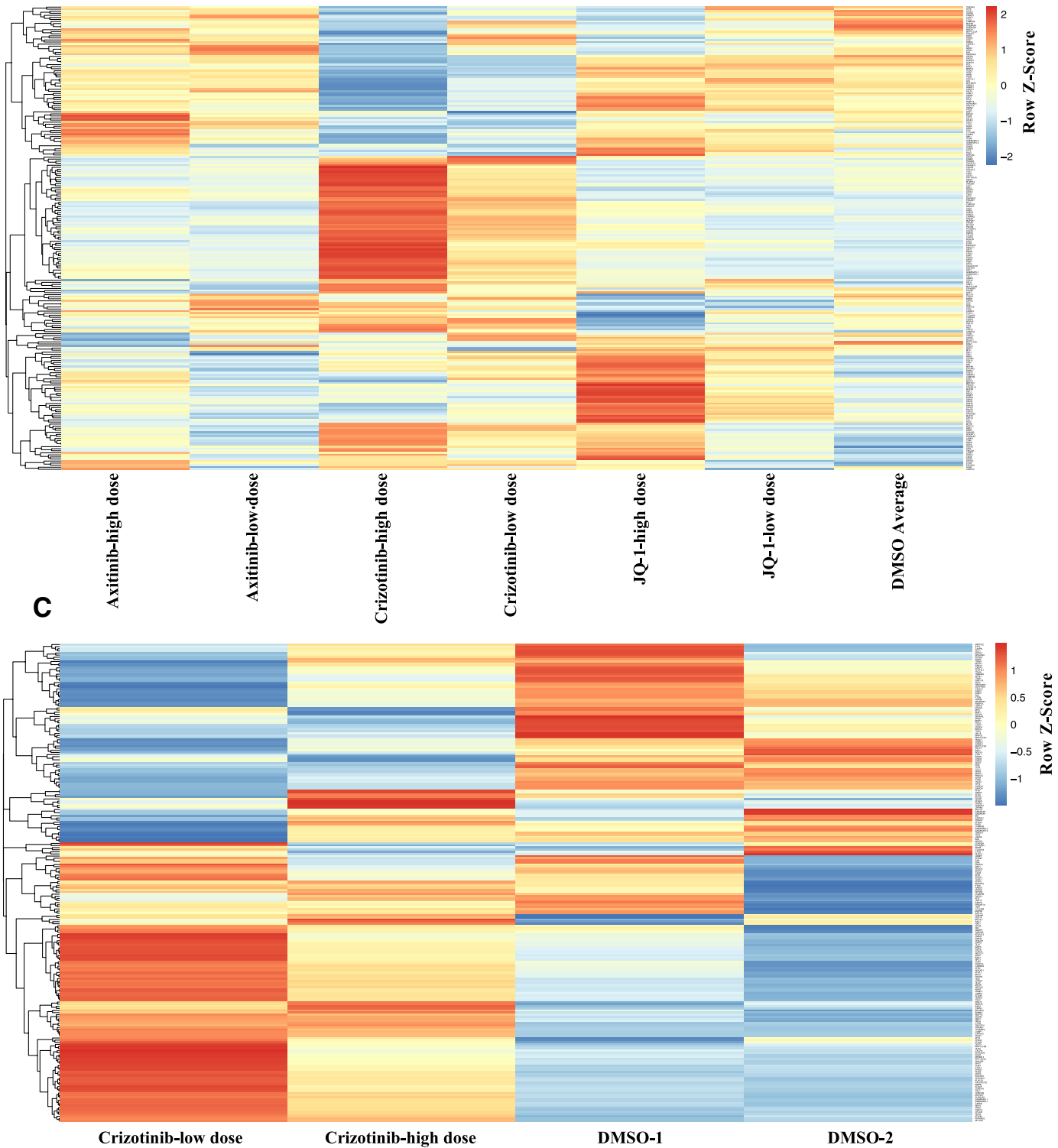
४Fig. 6 Gene profiling after compound treatments. A Identification of differentially expressed genes associated with axitinib, crizotinib, and JQ- 1 by volcano plots. The cutoff values, $\log 2$ of fold change $>1$ and $q<0.05$, were utilized to identify differentially expressed genes. Non-changed genes were shown in grey color. Red color was indicative of up-regulated genes and blue was indicative of down-regulated genes. $\mathbf{B}$ and $\mathbf{C}$, Analyses of expression profiles from RNA-seq data. After compound treatments, expression profiling analysis with RNAseq indicated that autophagy-related genes (ARGs) were frequently affected by treatment of crizotinib or JQ-1 (B), especially for crizotinib $(\mathbf{C})$

Supplementary Information The online version contains supplementary material available at https://doi.org/10.1007/s12094-021-02774-8.

Acknowledgements The authors thank the patients and their families and all investigators.

Funding This work was supported by the National Science and Technology Major Project of China (2016ZX09101094) and the National Natural Science Foundation of China (ID Number: 82072837).

Data availability The data in the current study are available from the corresponding authors on reasonable request.

\section{Declarations}

Conflict of interest The authors have declared no conflicts of interest.

Ethics approval The research study was reviewed and approved by the Domain-Specific Review Board, Cancer Hospital Chinese Academy of Medical Sciences (approval number: 20/246-2442, approval date: 2020-9-30).

Patient consent Written informed consents were obtained and patient details were anonymized before analysis.

Open Access This article is licensed under a Creative Commons Attribution 4.0 International License, which permits use, sharing, adaptation, distribution and reproduction in any medium or format, as long as you give appropriate credit to the original author(s) and the source, provide a link to the Creative Commons licence, and indicate if changes were made. The images or other third party material in this article are included in the article's Creative Commons licence, unless indicated otherwise in a credit line to the material. If material is not included in the article's Creative Commons licence and your intended use is not permitted by statutory regulation or exceeds the permitted use, you will need to obtain permission directly from the copyright holder. To view a copy of this licence, visit http://creativecommons.org/licenses/by/4.0/.

\section{References}

1. Komai Y, Fujiwara M, Fujii Y, Mukai H, Yonese J, Kawakami S, et al. Adult Xp11 translocation renal cell carcinoma diagnosed by cytogenetics and immunohistochemistry. Clin Cancer Res: Off J Am Assoc Cancer Res. 2009;15(4):1170-6.

2. Qu Y, Gu C, Wang H, Chang K, Yang X, Zhou X, et al. Diagnosis of adults Xp11.2 translocation renal cell carcinoma by immunohistochemistry and FISH assays: clinicopathological data from ethnic Chinese population. Sci Rep. 2016;6:21677.

3. Kauffman EC, Ricketts CJ, Rais-Bahrami S, Yang Y, Merino MJ, Bottaro DP, et al. Molecular genetics and cellular features of TFE3 and TFEB fusion kidney cancers. Nat Rev Urol. 2014;11(8):465-75.

4. Wang XT, Xia QY, Ye SB, Wang X, Li R, Fang R, et al. RNA sequencing of $\mathrm{Xp} 11$ translocation-associated cancers reveals novel gene fusions and distinctive clinicopathologic correlations. Modern pathol : Off United States Can Aca Pathol, Inc. 2018;31(9):1346-60.

5. Slade L, Pulinilkunnil T. The MiTF/TFE family of transcription factors: master regulators of organelle signaling, metabolism, and stress adaptation. Mol Cancer Res: MCR. 2017;15(12):1637-43.

6. Choo MS, Jeong CW, Song C, Jeon HG, Seo SI, Hong SK, et al. Clinicopathologic characteristics and prognosis of Xp11.2 translocation renal cell carcinoma: multicenter, propensity score matching analysis. Clin Genitourin Cancer. 2017;15(5):e819-25.

7. Garcia JG, Picken MM, Flanigan RC. The importance of histology and cytogenetics in decision making for renal cell carcinoma. World J Urol. 2008;26(2):155-60.

8. Qiu R, Bing G, Zhou XJ. Xp11.2 translocation renal cell carcinomas have a poorer prognosis than non-Xp11.2 translocation carcinomas in children and young adults: a meta-analysis. Int $\mathbf{J}$ Surg Pathol. 2010;18(6):458-64.

9. Grassi L, Alfonsi R, Francescangeli F, Signore M, De Angelis ML, Addario A, et al. Organoids as a new model for improving regenerative medicine and cancer personalized therapy in renal diseases. Cell Death Dis. 2019;10(3):201.

10. Fendler A, Bauer D, Busch J, Jung K, Wulf-Goldenberg A, Kunz S, et al. Inhibiting WNT and NOTCH in renal cancer stem cells and the implications for human patients. Nat Commun. 2020;11(1):929.

11. Lampis A, Carotenuto P, Vlachogiannis G, Cascione L, Hedayat S, Burke R, et al. MIR21 drives resistance to heat shock protein 90 inhibition in cholangiocarcinoma. Gastroenterology. 2018;154(4):1066-79.e5.

12. Puca L, Bareja R, Prandi D, Shaw R, Benelli M, Karthaus WR, et al. Patient derived organoids to model rare prostate cancer phenotypes. Nat Commun. 2018;9(1):2404.

13. Rao Q, Williamson SR, Zhang S, Eble JN, Grignon DJ, Wang $\mathrm{M}$, et al. TFE3 break-apart FISH has a higher sensitivity for Xp11.2 translocation-associated renal cell carcinoma compared with TFE3 or cathepsin K immunohistochemical staining alone: expanding the morphologic spectrum. Am J Surg Pathol. 2013;37(6):804-15.

14. Frick A, Fedoriw Y, Richards K, Damania B, Parks B, Suzuki O, et al. Immune cell-based screening assay for response to anticancer agents: applications in pharmacogenomics. Pharmgenomics Pers Med. 2015;8:81-98.

15. Hu S, Zhao Z, Yan H. Discovery and optimization of 5,7-dihydro-6H-pyrrolo[2,3-d]pyrimidin-6-one derivatives as mTORC1/ mTORC2 dual inhibitors. Bioorg Chem. 2019;92:103232.

16. Sachsenmeier KF, Hay C, Brand E, Clarke L, Rosenthal K, Guillard S, et al. Development of a novel ectonucleotidase assay suitable for high-throughput screening. J Biomol Screen. 2012;17(7):993-8.

17. Puca L, Bareja R, Prandi D, Shaw R, Benelli M, Karthaus WR, et al. Patient derived organoids to model rare prostate cancer phenotypes. (2041-1723 (Electronic)). Nat Commun. 2018;9:2404.

18. Zhang JH, Chung TD, Oldenburg KR. A simple statistical parameter for use in evaluation and validation of high throughput screening assays. J Biomol Screen. 1999;4(2):67-73.

19. De La Fuente R, Sonawane ND, Arumainayagam D, Verkman AS. Small molecules with antimicrobial activity against $E$. coli 
and $P$. aeruginosa identified by high-throughput screening. $\mathrm{Br} \mathrm{J}$ Pharmacol. 2006;149(5):551-9.

20. Scholle MD, Liu C, Deval J, Gurard-Levin ZA. Label-free screening of SARS-CoV-2 NSP14 exonuclease activity using SAMDI mass spectrometry. SLAS Discov : Adv Life Sci R \& D. 2021;26(6):766-74.

21. Zhang XD, Espeseth AS, Johnson EN, Chin J, Gates A, Mitnaul $\mathrm{LJ}$, et al. Integrating experimental and analytic approaches to improve data quality in genome-wide RNAi screens. J Biomol Screen. 2008;13(5):378-89.

22. Shi Y, Liu X, Fredimoses M, Song M, Chen H, Liu K, et al. FGFR2 regulation by picrasidine $\mathrm{Q}$ inhibits the cell growth and induces apoptosis in esophageal squamous cell carcinoma. J Cell Biochem. 2018;119(2):2231-9.

23. Schroeder RD, Choi W, Hong DS, McConkey DJ. Autophagy is required for crizotinib-induced apoptosis in MET-amplified gastric cancer cells. Oncotarget. 2017;8(31):51675-87.

24. Sakamaki JI, Wilkinson S, Hahn M, Tasdemir N, O'Prey J, Clark $\mathrm{W}$, et al. Bromodomain protein BRD4 is a transcriptional repressor of autophagy and lysosomal function. Mol Cell. 2017;66(4):51732.e9.

25. Solano C, Thapa S, Chisti MM. Adult Xp11.2 translocation renal cell carcinoma managed effectively with pazopanib. BMJ Case Rep. 2021;14(6):e243058.

26. Broutier L, Mastrogiovanni G, Verstegen MM, Francies HE, Gavarró LM, Bradshaw CR, et al. Human primary liver cancerderived organoid cultures for disease modeling and drug screening. Nat Med. 2017;23(12):1424-35.

27. Liu HD, Xia BR, Jin MZ, Lou G. Organoid of ovarian cancer: genomic analysis and drug screening. Clin Transl Oncol: Off Publ Fed Spanish Oncol Soc Natl Cancer Inst Mexico. 2020;22(8):1240-51.

28. Vlachogiannis G, Hedayat S, Vatsiou A, Jamin Y, FernándezMateos J, Khan K, et al. Patient-derived organoids model treatment response of metastatic gastrointestinal cancers. Science (New York, NY). 2018;359(6378):920-6.

29. Czerniecki SM, Cruz NM, Harder JL, Menon R, Annis J, Otto EA, et al. High-throughput screening enhances kidney organoid differentiation from human pluripotent stem cells and enables automated multidimensional phenotyping. Cell Stem Cell. 2018;22(6):929-40.e4.

30. Park I, Lee SH, Lee JL. A multicenter phase II trial of axitinib in patients with recurrent or metastatic non-clear-cell renal cell carcinoma who had failed prior treatment with temsirolimus. Clin Genitourin Cancer. 2018;16(5):e997-1002.

31. Rodig SJ, Shapiro GI. Crizotinib, a small-molecule dual inhibitor of the c-Met and ALK receptor tyrosine kinases. Curr Opin Investig Drugs. 2010;11(12):1477-90.

32. Dey A, Nishiyama A, Karpova T, McNally J, Ozato K. Brd4 marks select genes on mitotic chromatin and directs postmitotic transcription. Mol Biol Cell. 2009;20(23):4899-909.

33. Ploper D, Taelman VF, Robert L, Perez BS, Titz B, Chen HW, et al. MITF drives endolysosomal biogenesis and potentiates Wnt signaling in melanoma cells. Proc Natl Acad Sci USA. 2015;112(5):E420-9.

34. Bahrami A, Bianconi V, Pirro M, Orafai HM, Sahebkar A. The role of TFEB in tumor cell autophagy: diagnostic and therapeutic opportunities. Life Sci. 2020;244:117341.

35. Sun G, Chen J, Liang J, Yin X, Zhang M, Yao J, et al. Integrated exome and RNA sequencing of TFE3-translocation renal cell carcinoma. Nat Commun. 2021;12(1):5262.

36. Megiorni F, McDowell HP, Camero S, Mannarino O, Ceccarelli S, Paiano M, et al. Crizotinib-induced antitumour activity in human alveolar rhabdomyosarcoma cells is not solely dependent on ALK and MET inhibition. J Exp Clin Cancer Res. 2015;34:112.

37. Espinos E, Lai R, Giuriato S. The dual role of autophagy in crizotinib-treated ALK (+) ALCL: from the lymphoma cells drug resistance to their demise. Cells. 2021;10(10):2517.

Publisher's Note Springer Nature remains neutral with regard to jurisdictional claims in published maps and institutional affiliations. 